\title{
A Simplified Approach for Provisionalization Utilizing Extracted Natural Tooth as a Pontic-A Clinical Report
}

\author{
Ankita Rathi, MDS ${ }^{\text {a }}$, Suraj Ram Bhakta Mathema, MDS ${ }^{\mathrm{b}}$ \\ ${ }^{a}$ Lecturer, Department of Prosthodontics, Nobel Medical College and Teaching Hospital \\ ${ }^{\mathrm{b}}$ Professor and Head of Department, Department of Prosthodontics \\ Peoples Dental College and Teaching Hospital, Nepal. \\ drankitarathi@yahoo.com
}

\begin{abstract}
The loss of anterior teeth due to trauma, caries, and periodontal diseases can result in serious functional and esthetic disabilities compromising the patients' quality of life. The current scenario in dentistry proposes several treatment modalities for the esthetic and functional replacement of a missing anterior tooth which includes implant supported single crown, conventional FPD, Resin Bonded FPD or RPD. However when certain clinical conditions or unwillingness of the patient preclude the replacement of missing tooth with any of these prostheses, a more conservative treatment modality in the form of fiber reinforce composite resin fixed partial denture with composite resin, porcelain fused to metal, all ceramic or natural tooth pontic can be considered as a definitive treatment alternative.
\end{abstract}

Keywords: extracted tooth, fixed provisional, esthetic

\section{INTRODUCTION}

Anterior teeth play an important role in overall appearance. The loss of anterior teeth may be due to trauma, caries, periodontal disease, root resorption, or failed endodontic treatment which can be considered as emergency and often requires replacement of the tooth in a single visit. ${ }^{1,2}$ Clinicians are concerned for the patient's esthetics and function but also must realize that the process of rehabilitation can be undertaken with a series of complex procedures that could take several months to complete. ${ }^{3}$

There are many options documented for the purpose of provisionalizing anterior teeth during the surgical healing phases of treatment. ${ }^{3}$ Conventional acrylic removable partial dentures (RPDs) are most commonly preferred due to financial reasons. Acrylic RPD when placed immediately after the tooth is extracted, may be uncomfortable for the patient, and also impede healing process. They also fail to preserve the extraction socket, which results in an objectionable loss of soft and hard tissues in the extraction area. ${ }^{4}$

Fixed provisional restoration using extracted natural tooth, acrylic denture tooth, or composite resin tooth as a pontic embedded in wire, glass fibre, metal mesh, nylon, or cast metal frameworks has been described, but these can result in poor bonding at the interface between composite and metal or nylon, leading to failure of the restoration. The introduction of bondable fiber (i.e., polyethylene or quartz)permits for placement of periodontal splint as well as bonded resin-based, single-visit fixed partial dentures (FPDs). ${ }^{5-7}$

The use of patient's extracted tooth as a pontic has been reported in the literature, with advantages of ideal shape, contour, color of the surrounding dentition and instant availability for bonding during surgery, with minimum necessity for preliminary lab work. ${ }^{4,8} \mathrm{~A}$ fixed natural tooth pontic extends into the extraction socket in order to shape the soft tissue and support the adjacent papilla so that the final restoration emerges from the implant platform with the same contour and dimensions as the natural tooth..$^{9-11}$ 
A Simplified Approach for Provisionalization Utilizing Extracted Natural Tooth as a Pontic-A Clinical Report

This clinical report presents a practical technique for immediate interim tooth replacement utilizing the clinical crown to assist the clinician in providing an esthetically acceptable treatment option.

\section{Clinical RePort}

A 32-year-old male patient presented to the Department of Prosthodontics and Maxillofacial Prosthetics with the complaint of mobile right maxillary front tooth for 2 weeks. The patient gave a history of traumatic injury to maxillary anterior region 2 weeks ago while playing football. On clinical examination, tooth \#11 showed grade 3 mobility (Fig. 1). A radiographic examination revealed horizontal fracture of \#11 in the middle third of the root with severe bone loss with respect to \#11 (Fig. 2).

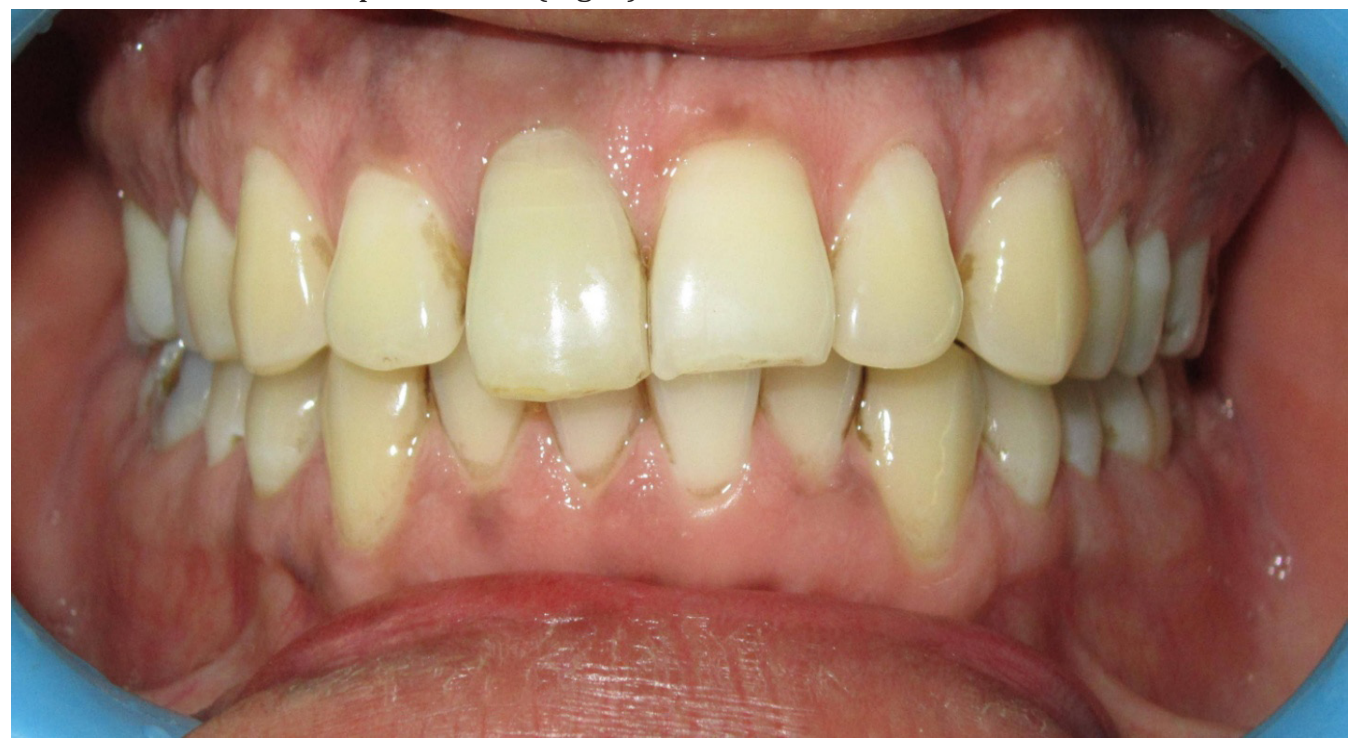

Fig1. Pre treatment intraoral photograph of the patient

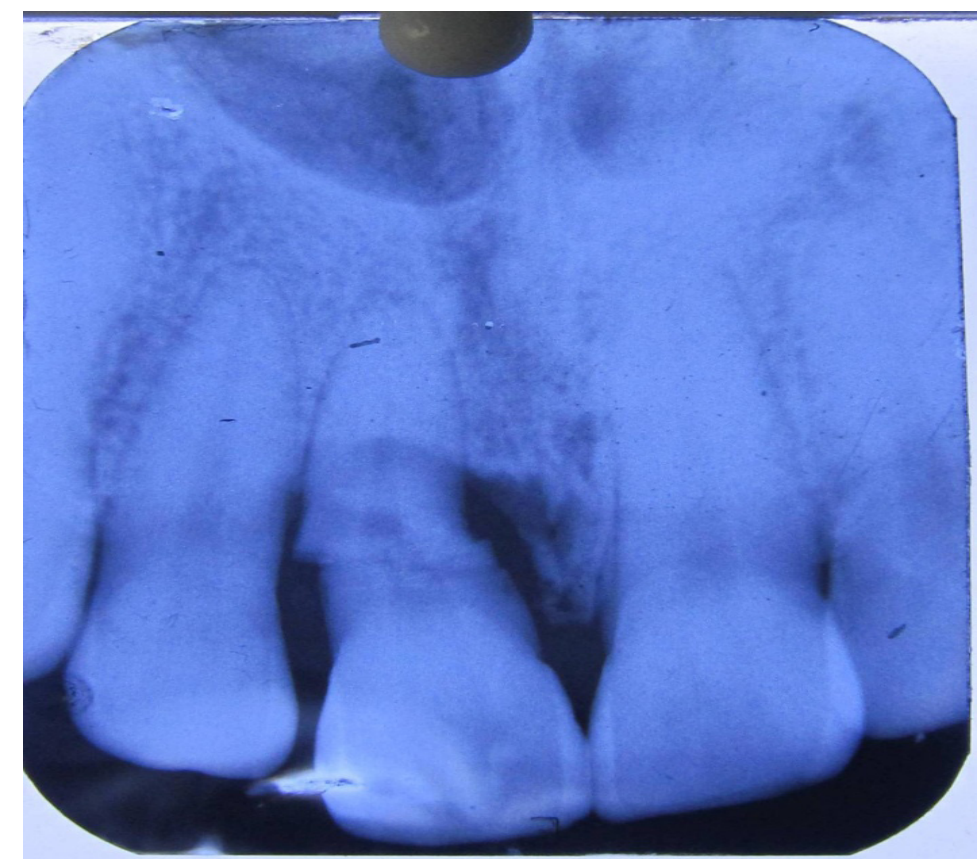

Fig2. Intraoral periapical radiograph of 11 and 21 
A Simplified Approach for Provisionalization Utilizing Extracted Natural Tooth as a Pontic-A Clinical Report

Since the prognosis of \#11 was poor, it was extracted (Fig 3 and Fig 4). The extraction socket was curetted to remove the granulation tissue, followed by placement of a freeze-dried bone graft(osseograft) and a collagen membrane (Colo Gide) to permit guided bone regeneration. Surgical closure was done with 3-0 catgut suture.

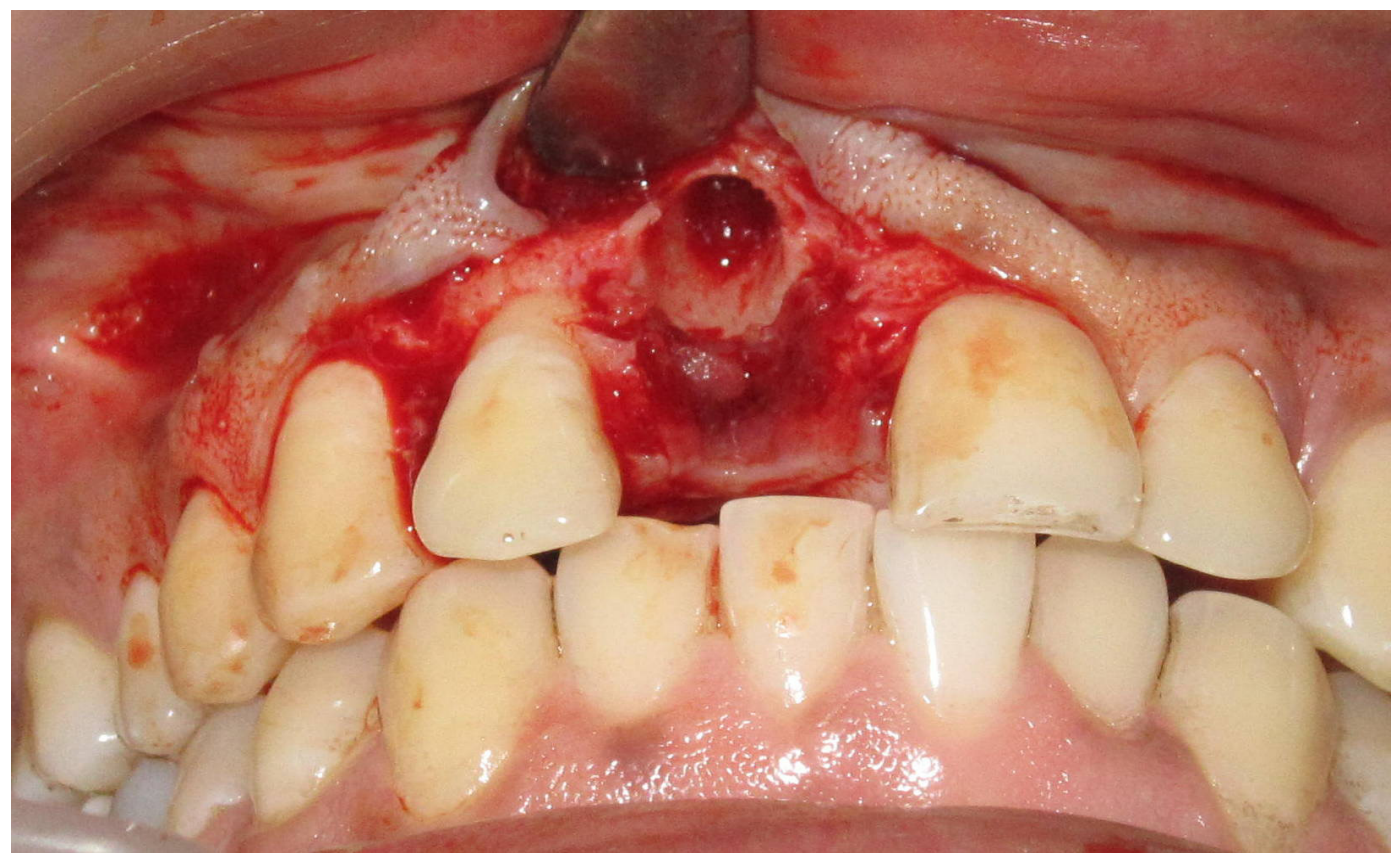

Fig3. Post Extraction of 11

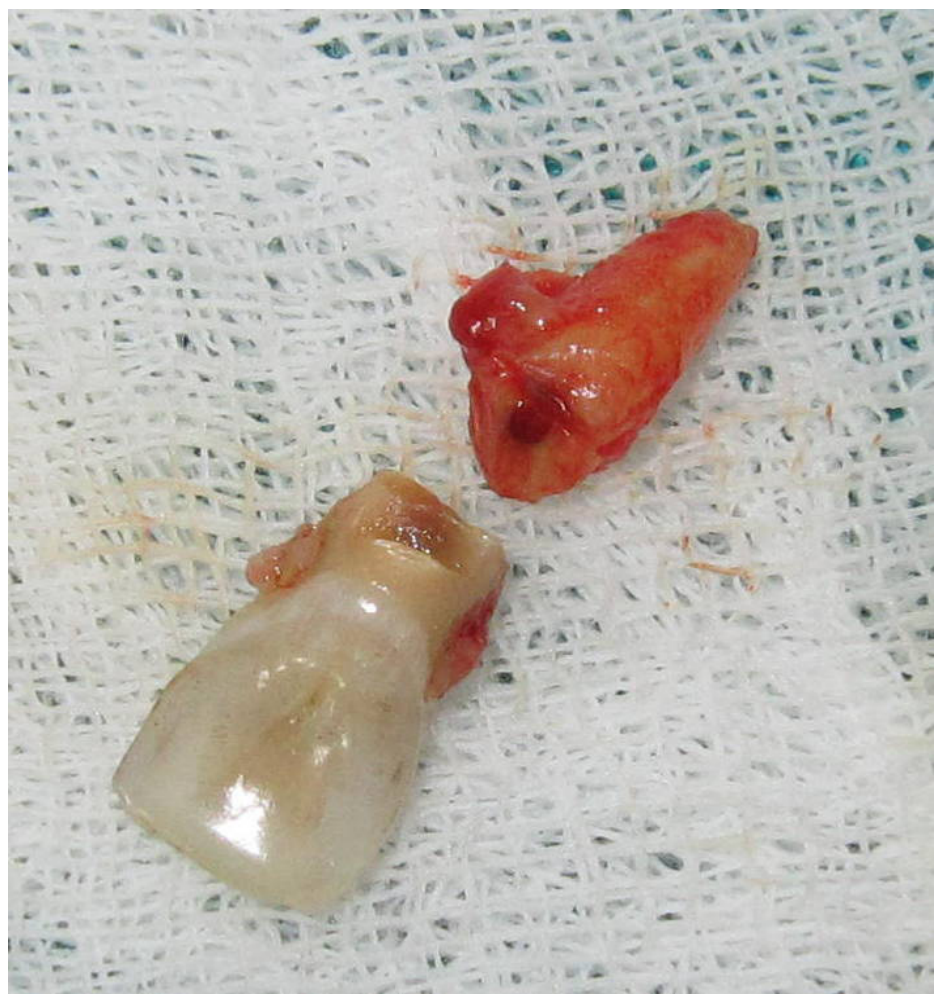

Fig4. extracted tooth to be used as tooth pontic 
In order to achieve an ideal gingival architecture with properly supported papilla, an interim prosthesis was fabricated using the patient's extracted natural teeth. The pulp tissue of the extracted tooth was removed. The canal was cleaned, and light-cure composite resin was cured within the canal. A modified ridge lap design was given to the natural tooth pontic, satisfying both oral hygiene and esthetics. A 2-mm recess groove was made into the pontic for placement of the quartz fiber teeth. Adjacent teeth were etched, bonded, and light-cured. Quartz fiber soaked in adhesive was bonded to adjacent teeth flowable composite. The groove in the natural tooth pontic was filled with flowable composite, seated on the fiber, and light cured. The occlusion was checked and adjusted with a fine diamond, and the entire lingual connector was polished with silicone points and cups. Excess interproximal resin was removed with fine-tipped composite finishing diamonds (Fig 5 and Fig 6).

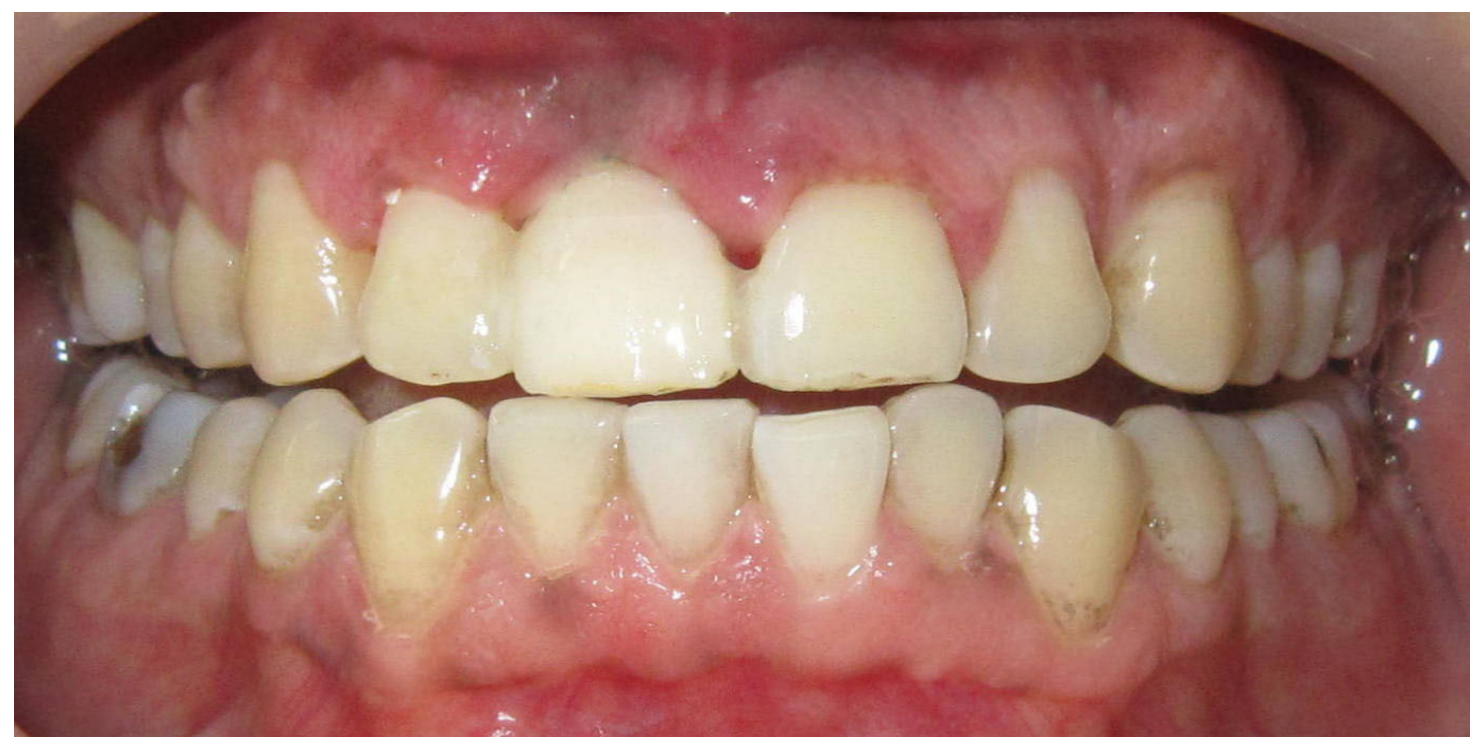

Fig5. Post treatment photograph - frontal view

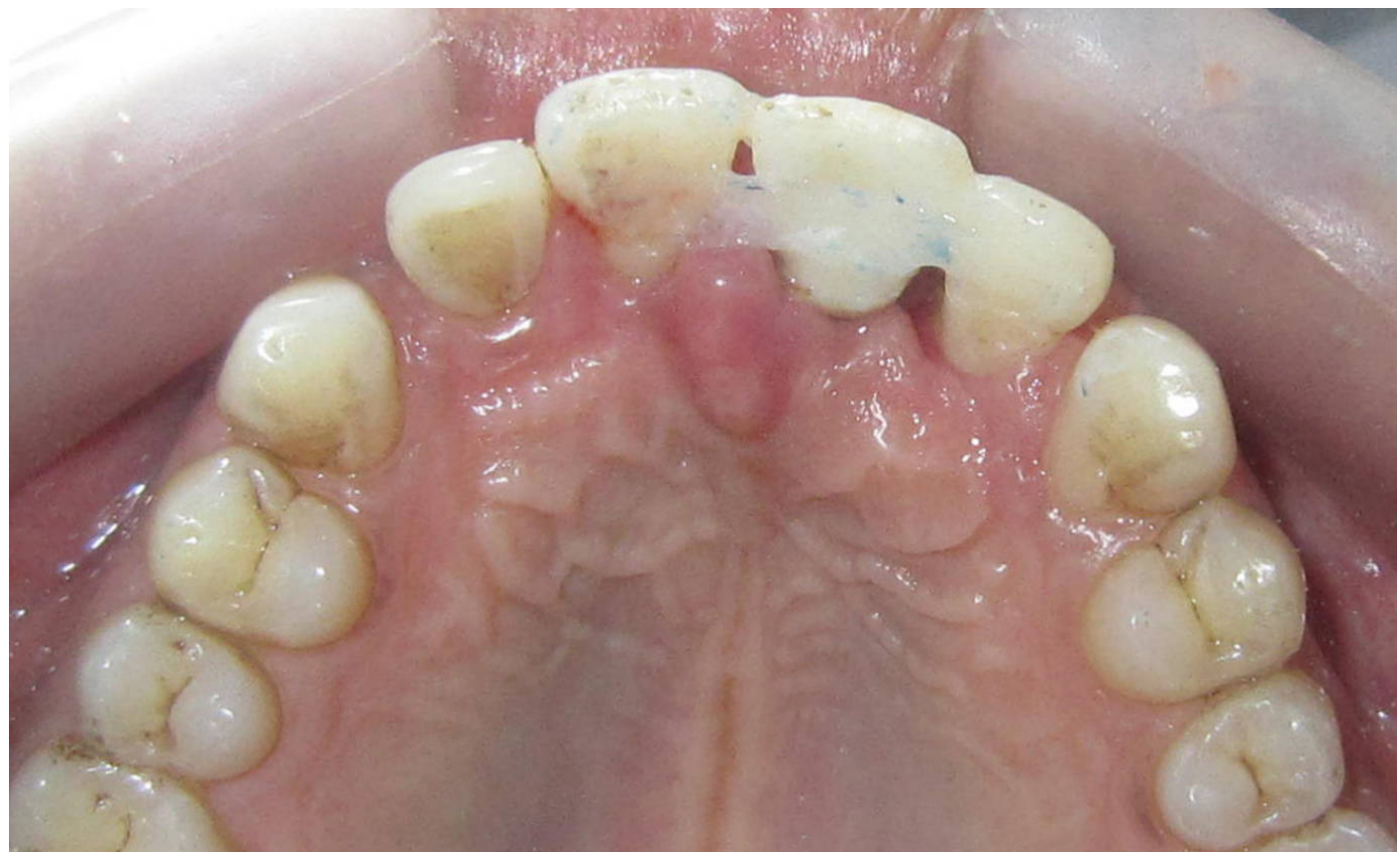

Fig6. Post treatment photograph -occlusal view 
A Simplified Approach for Provisionalization Utilizing Extracted Natural Tooth as a Pontic-A Clinical Report

Follow up was done after 1 week, 1 month, and 3 months. The healing was uneventful. The case is planned for implant placement in respect to 11 after 6 months of followup.

\section{DiscusSiON}

Replacement of the missing anterior teeth with an artificial substitute of same shape, shade and size is a challenging task, especially when the patient has high esthetic demands and needs an immediate replacement

Different prosthetic rehabilitation options are available for single-visit replacement of missing teeth in the esthetic zone during surgical healing phase. ${ }^{12}$ An acrylic partial denture is an acceptable, affordable, and convenient method of provisionalization, but its bulkiness may impede healing. After surgical extraction of tooth and root fragments, placement of bone and CT grafts, it is imperative to not place pressure on the surgical site and to avoid the potential displacement of fragile soft-tissue architecture. Acrylic partial dentures are usually tissue-borne prosthesis and lack hard-tissue stops to prevent apical forces in the area of the singletooth site. Therefore they should be avoided. ${ }^{13,14}$

A non-removable treatment option, such as resin bonded FPD offers several advantages such as enhanced esthetics and ease of use. This approach would also permit utilization of a patient's natural crown as a pontic for an immediate FPD with little or no need to perform complicated laboratory procedure.

A resin bonded fixed partial denture (RBFPD) is retained and supported by adjacent teeth, and thus remains passive over the surgical site.

Cast-metal-reinforced RBFPDs were originally developed as a conservative option for definitive tooth replacement, but are frequently used as interim prostheses for implant patients. However, optimal esthetics may be a problem with this type of prosthesis because thin or translucent teeth are often unable to mask the gray color of the palatal metal retainers. ${ }^{15}$ The concept of using an extracted natural tooth pontic is predicated on the ability to section the clinical crown and desired amount of root structure at the ideal length to maintain support for the interproximal papilla without displacing or disrupting CT or osseous grafts. To ensure that the natural tooth pontic remains stable throughout the course of treatment, it must be securely attached extracoronally (without preparation) to the adjacent teeth. ${ }^{12}$

Ideally, the adjacent teeth should be intact and periodontally sound, with ideal interproximal contact to the extracted natural tooth pontic. The functional occlusal stress on the pontic site should be minimal. Preoperative centric, working, and nonworking contacts must be assessed to determine whether displacing forces can be reduced or eliminated. ${ }^{13}$

One major advantage of retaining the patient's natural crown is that the patient can better tolerate the effect of tooth loss. Also the pontic has the right size, shape, and color. The emergence profile and gingival contour can be achieved. The position, size, and shape of the interdental papilla are maintained. The natural tooth does not interfere with the healing of the socket. It also prevents the collapse of the papilla. Cervical bone configuration maintains esthetics. Labial plate of bone is not compressed and is maintained in its natural position, helping to give a better labial profile to the restoration. ${ }^{12}$

In the past, number of different techniques have been described in the literature for splinting teeth and adding a natural tooth pontic, denture tooth, or composite resin tooth pontic with adhesive composite resins, wire, metal mesh, nylon, mesh, and cast metal frameworks. ${ }^{4}$ The inherent problems with these materials were their inability to be chemically incorporated into the dental resin. Also these materials could not support the repeated loading stresses placed on the FPD during normal and para-function. The problem associated with the placement of composite resin splints with submerged wires and mesh grids was that more bulk and thickness of composite resin was necessary to protect against breakage. This over-bulking of the restoration led to an increase in food

American Research Journal of Dentistry

Page 20 
A Simplified Approach for Provisionalization Utilizing Extracted Natural Tooth as a Pontic-A Clinical Report

and plaque retention, resulting in making it more difficult to clean around the restoration and maintain good oral health. ${ }^{16,17}$ The challenge to place a thin but strong bonded composite resin-based, single-visit FPD was met with the introduction of a high-strength polyethylene, bondable, biocompatible, esthetic, easily manipulated, fiber ribbon that could be embedded into a resin structure. ${ }^{18}$

\section{CONCLUSION}

Provisionalization in the aesthetic zone following extraction and bone graft procedure is often overlooked in terms of the health of underlying tissues. Therefore, the provisional restoration provided should restore optimum esthetics without hampering health implant bed. Thus, Clinicians need to be aware of the range of techniques and materials for short-, medium-, and long-term provisionalization depending on the clinical condition.

\section{REFERENCE}

1. Strassler HE, Taler D, and Sensi LG. Fiber Reinforcement for One-Visit Single-Tooth Replacement. Dent Today. June 2007; 26(6):120, 122-5.

2. Lathi $\mathrm{S}$ et al. Fractured tooth- immediate aesthetic solution- a case report Volume 4 Issue 4 Jul.-Aug.2014 international journal of research in dentistry

3. Blank J T. A Novel Approach for a Single-Tooth Provisional. Dent Today. 2012 Apr;31(4):134, 136-9.

4. S Bhargava, Ri Namdev, S Dutta,. Immediate fixed temporization with a natural tooth crown pontic following failure of replantation. Contemp Clin Dent. 2011 Jul-Sep; 2(3): 226-229

5. Ibsen RL. One-appointment technic using an adhesive composite. Dental survey. 1973 Feb;49(2):30.

6. Jordan RE, Suzuki M, Sills PS, Gratton DR, Gwinnett JA. Temporary fixed partial dentures fabricated by means of the acid-etch resin technique: a report of 86 cases followed for up to three years. The Journal of the American Dental Association. 1978 Jun 1;96(6):994-1001.

7. Miller TE, Barrick JA. Pediatric trauma and polyethylene reinforced composite fixed partial denture replacements: a new method. Journal (Canadian Dental Association). 1993 Mar;59(3):252.

8. Rudraprasad CA, Nandeeshwa DB. Natural tooth pontic: A temporary immediate provisional for a difficult esthetic situation. J indprosthsoc. 2008;8:122-5.

9. Kois JC. Altering gingival levels: The restorative connection, Part I: Biologic variables. J Esthet Restor Dent. 1994;6:3-7.

10. Banerjee R, Banerjee S, Usha R. Ovate pontic design: An aesthetic solution to anterior missing tooth-A case report. Journal of Clinical and Diagnostic Research. 2010;4:2996-2999.

11. Mishra N, Singh BP, Rao J, et al. Improving prosthetic prognosis by connective tissue ridge augmentation of alveolar ridge. Indian J Dent Res. 2010;21:129-131.

12. Lathi S, Lathi A, Sonambekar A, Bhawsar S, Sonambekar A, Lathi A. Fractured tooth-immediate aesthetic solution-a case report. International Journal of Clinical Dentistry. 2015 Jul 1;8(3).

13. Blank JT. A novel approach for a single-tooth provisional. Dentistry today. 2012 Apr;31(4):134-6.

14. Santosa RE. Provisional restoration options in implant dentistr. Australian dental journal. 2007 Sep 1;52(3):234-42. 
A Simplified Approach for Provisionalization Utilizing Extracted Natural Tooth as a Pontic-A Clinical Report

15. Cho S, Shetty S, Froum S, Elian N, Tarnow D. Fixed and removable provisional options for patients undergoing implant treatment. Compendium-Newtown-. 2007 Nov 28;28(11):604.

16. Strassler HE, Haeri A, Gultz JP. New-generation bonded reinforcing materials for anterior periodontal tooth stabilization and splinting. Dent Clin North Am. 1999;4:105-26.

17. 7. Pollack RP. Non-crown and bridge stabilization of severely mobile, periodontally involved teeth.A 25year perspective. Dent Clin North Am. 1999;43:77-103.

18. Strassler HE, Taler D, Sensi L. Single visit natural tooth pontic bridge with fiber reinforcement ribbon. Oral Health J. 2007:24-36.

Citation: Ankita Rathi, MDS, Suraj Ram Bhakta Mathema, MDS, "A Simplified Approach for Provisionalization Utilizing Extracted Natural Tooth as a Pontic-A Clinical Report". American Research Journal of Dentistry; 1(1): 16-22.

Copyright (c) Ankita Rathi, MDS, Suraj Ram Bhakta Mathema, MDS, This is an open access article distributed under the Creative Commons Attribution License, which permits unrestricted use, distribution, and reproduction in any medium, provided the original work is properly cited. 